Research Paper

\title{
A Targetable Molecular Chaperone Hsp27 Confers Aggressiveness in Hepatocellular Carcinoma
}

\author{
Yurong Zhang ${ }^{*}$, Xuemei Tao ${ }^{{ }^{*}}$, Guangzhi Jin ${ }^{2 *}$, Haojie Jin ${ }^{1}$, Ning Wang ${ }^{1}$, Fangyuan $\mathrm{Hu}^{1}$, Qin Luo ${ }^{1}$, Huiqun \\ Shu1 ${ }^{1}$, Fangyu Zhao ${ }^{1}$, Ming Yao ${ }^{1}$, Jingyuan Fang ${ }^{1}$, Wenming Cong${ }^{2}$, Wenxin Qin ${ }^{1}{ }^{\bowtie}$, Cun Wang ${ }^{\bowtie}$ \\ 1. State Key Laboratory of Oncogenes and Related Genes, Shanghai Cancer Institute, Renji Hospital, Shanghai Jiao Tong University School of Medicine, \\ Shanghai, China. \\ 2. Department of Pathology, Eastern Hepatobiliary Surgery Hospital, Second Military Medical University. \\ * These authors contribute equally to this work. \\ $\triangle$ Corresponding authors: Cun Wang; Mailing Address: State Key Laboratory of Oncogenes and Related Genes, Shanghai Cancer Institute, Renji Hospital, \\ Shanghai Jiao Tong University School of Medicine, No. 25/Ln 2200 Xie-Tu Road, Shanghai 200032, China; Tel: +86-21-64436572; Fax: +86-21-64432142; Email: \\ cwang@shsci.org. Or Wenxin Qin; Mailing Address: State Key Laboratory of Oncogenes and Related Genes, Shanghai Cancer Institute, Renji Hospital, Shanghai \\ Jiao Tong University School of Medicine, No. 25/Ln 2200 Xie-Tu Road, Shanghai 200032, China; Tel: +86-21-64436581; Fax: +86-21-64432142; Email: \\ wxqin@sjtu.edu.cn.
}

( ) Ivyspring International Publisher. Reproduction is permitted for personal, noncommercial use, provided that the article is in whole, unmodified, and properly cited. See http://ivyspring.com/terms for terms and conditions.

Received: 2015.12.14; Accepted: 2016.01.11; Published: 2016.02.17

\begin{abstract}
Heat shock protein 27 (Hsp27) is an ATP-independent molecular chaperone and confers survival advantages and resistance to cancer cells under stress conditions. The effects and molecular mechanisms of Hsp27 in HCC invasion and metastasis are still unclear. In this study, hepatocellular carcinoma $(\mathrm{HCC})$ tissue array $(n=167)$ was used to investigate the expression and prognostic relevance of $\mathrm{Hsp} 27$ in $\mathrm{HCC}$ patients. $\mathrm{HCC}$ patients with high expression of Hsp27 exhibited poor prognosis. Overexpression of Hsp27 led to the forced invasion of HCC cells, whereas silencing $\mathrm{Hsp} 27$ attenuated invasion and metastasis of HCC cells in vitro and in vivo. We revealed that $\mathrm{Hsp} 27$ activated Akt signaling, which in turn promoted MMP2 and ITGA7 expression and HCC metastasis. We further observed that targeting Hsp27 using OGX-427 obviously suppressed HCC metastasis in two metastatic models. These findings indicate that $\mathrm{Hsp} 27$ is a useful predictive factor for prognosis of HCC and it facilitates HCC metastasis through Akt signaling. Targeting Hsp27 with OGX-427 may represent an attractive therapeutic option for suppressing HCC metastasis.
\end{abstract}

Key words: Hsp27, hepatocellular carcinoma, metastasis, prognosis, OGX-427.

\section{Introduction}

Liver cancer is one of the most frequently diagnosed malignancies and the third leading cause of cancer-related deaths worldwide, with an estimated 782,500 annual diagnoses and 745,500 annual deaths during 2012 [1]. China accounts for about $50 \%$ of the total new diagnosed cases and related deaths. More than $80 \%$ of primary liver cancers occurring worldwide are hepatocellular carcinoma (HCC) [1]. HCC tumors are often resistant to chemotherapy, so surgical interventions including surgical resection and liver transplantation are the current standard practices for the treatment of HCC [2]. Nonetheless, approximately only $30-40 \%$ of HCC patients are suitable for curative treatment at the time of diagnosis [3,4]. And the overall survival rate following surgical interventions remains unsatisfactory due to high rates of recurrence and metastasis $[5,6]$. Sorafenib treatment was established as the standard therapy for patients with advanced HCC [7]. However, this promising systemic therapy has indicated limited survival benefits with very low rates of tumor response [8]. Currently, mechanisms underlying metastasis and recurrence remain poorly understood. It is urgent to further clarify the mechanism underlying metastasis and identify potential therapeutic targets for HCC treatment.

Heat shock proteins (Hsps) are highly conserved 
molecular chaperones, which are essential components of diverse cell types [9-11]. They are often expressed at low levels under normal conditions, but significantly up-regulated in response to cellular stresses [10,11]. Malignant cells are constantly subjected to stress conditions, including the existence of ischemic, hypoxic and acidic microenvironment, exposure to cytotoxic agents or radiotherapy, and genomic instability. Up-regulation of Hsps is a key driver of adaptive stress-response pathways in many malignancies, which could help cancer cells adapt to different stress conditions [10,11]. Hsps are often classified according to their molecular weights, including Hsp27, Hsp40, Hsp60, Hsp70, Hsp90, Hsp110, etc. There are also several other proteins with Hsp-like properties such as clusterin which are discussed with these small Hsps [12-14]. Various inhibitors of Hsps have been developed and proposed as possible strategies for cancer therapy. It is reasonable that targeting Hsps with specific inhibitor alone or in combination with other chemotherapy agents may hold promise for the improvement of outcomes for cancer patients $[10,11]$.

Hsp27 is overexpressed in many types of human cancers including prostate cancer [15], ovarian cancer [16], gastric cancer [17], and breast cancer [18]. Hsp27 is an important chaperon molecule, which is responsible for modulating more than 200 client proteins, including translationally controlled tumor protein (TCTP) [19], cytochrome-c [20], caspase-3 [21], and $14-3-3 \zeta$ [22], etc. A recent study reported that a total of 226 proteins were identified as Hsp27-interacting proteins using a yeast two-hybrid approach [23]. Hsp27 client proteins are involved in almost all aspects of cancer initiation and development, including translation initiation, cytoskeleton organization, apoptosis inhibition, invasion, cell cycle regulation, DNA repair, RNA splicing, and modulation of signal transduction [23]. These diverse cellular functions of Hsp27 may be due to its different associated partner proteins. Emerging studies reveal that Hsp27 is often induced by chemotherapies or targeted therapies. It confers resistant property of several cancer types. Inhibition of the expression of Hsp27 with siRNA or OGX-427, an antisense oligonucleotide in phase II trials, synergistically enhanced the efficacy of EGFR tyrosine kinase inhibitor [24], radiation [25], Hsp90 inhibitor [26], MET inhibitor [27], paclitaxel [28]. and gemcitabine [29].

It has also been described that Hsp27 functions as a prometastatic protein in different cancer types and is closely related with the aggressiveness of tumor behavior, metastasis and poor prognosis [30,31]. Hsp27 regulates the EMT process and NF-kB activity to maintain breast cancer stem cells [32]. Targeting
Hsp27 interferes with bone metastasis of breast cancer in vivo [33]. Hsp27 may facilitate metastatic potential of head and neck squamous cell cancer cells [34]. In addition, Hsp27 is required for EGF, HGF and IL-6 induced EMT in prostate cancer [35-37]. Hsp27 suppression by OGX-427 obviously inhibits metastasis in prostate cancer [36]. In HCC, a previous study indicated that Hsp27 was involved in PKC beta-mediated HCC cell motility and invasion [38]. However, detailed mechanisms of Hsp27 in HCC metastasis are still unclear. Furthermore, the potential of Hsp27 as a therapeutic target for HCC metastasis will need to be further studied.

In this study, we investigated the clinicopathologic significance of Hsp27 expression and explored the underlying molecular mechanism of Hsp27-mediated invasion and metastasis in HCC. Additionally, the therapeutic effect of Hsp27 inhibitor (OGX-427) in suppressing HCC metastasis was also addressed.

\section{Materials and Methods}

\section{Cell culture}

The human HCC cell lines, HepG2 and SK-Hep1, were purchased from the American Type Culture Collection (ATCC, VA, USA). HCC cell line SMMC7721 was purchased from Shanghai Institute of Cell Biology, Chinese Academy of Sciences. HCC cell lines with stepwise metastatic potential (MHCC97L, MHCC97H and HCCLM3) were established by Liver Cancer Institute of Fudan University. Huh7 cells were purchased from Riken Cell Bank (Tsukuba, Japan). All the cell lines were cultured in Dulbecco's Modified Eagle's Medium (DMEM) supplemented with 10\% fetal bovine serum (FBS) and antibiotics $(100 \mathrm{U} / \mathrm{ml}$ penicillin, $100 \mathrm{mg} / \mathrm{ml}$ streptomycin), in a $5 \% \mathrm{CO} 2$ atmosphere at $37^{\circ} \mathrm{C}$.

\section{Quantitative real-time PCR}

Real-time PCR analyses were performed according to the manufacturer's instructions (TaKaRa Biotechnology). The primers used are given in Supplementary Table S1. The expression levels were normalized against the internal reference gene $\beta$-actin, and the relative expression levels were displayed using the $2^{-\Delta \Delta \mathrm{Ct}}$ method.

\section{Western blotting}

Western blot analysis was performed as previously described [13]. Briefly, Proteins were separated by SDS-PAGE and transferred onto PVDF membrane. Membranes were incubated with the specific primary antibodies and then with HRP-conjugated secondary antibodies. Protein bands were visualized using chemiluminescence detection. The following anti- 
bodies were used: antibodies to Anti-Hsp27 (1:1000, Cell Signaling Technology), $\beta$-actin (1:25000, Sigma), GAPDH (1:5000, Sigma), Akt (1:1000, Cell Signaling Technology), p-Akt (1:1000, Cell Signaling Technology).

\section{HCC specimens and immunohistochemical staining}

HCC specimens were obtained from 167 patients who underwent curative surgery in Eastern Hepatobiliary Hospital of the Second Military Medical University. Patients were not subjected to any pre-operative anti-cancer treatment. Ethical approval was obtained from the Eastern Hepatobiliary Hospital Research Ethics Committee, and written informed consent was obtained from each patient. Overall survival (OS) was defined as the length of time between the surgery and death. The time to recurrence (TTR) was defined as the interval between the dates of surgery and recurrence. Patients were censored on the date of the last follow-up or death, if recurrence was not diagnosed. Immunohistochemical staining for Hsp27 was done as previously described [13]. Immunohistochemical score was independently assessed by 2 pathologists without knowledge of patient characteristics. The percentage of positive cells was divided into 4 grades: $0-25 \%, 1 ; 26 \%-50 \%, 2 ; 51 \%-75 \%, 3$; and $>75 \%, 4$. The intensity of the staining was divided into 4 grades (intensity scores): no staining, 0; light brown, 1; brown, 2; and dark brown, 3. The immunostaining score was evaluated on the basis of percentage score $\times$ intensity score. Overall scores of $0-3,4-6,7-9$, or $10-12$ were defined as negative $(-)$, weak $(+)$, moderate $(++)$, or strong $(+++)$ staining, respectively.

\section{Immunofluorescent staining}

HCC cells were grown on glass coverslips. After an attachment period of $24 \mathrm{~h}$, cells were fixed with $4 \%$ paraformaldehyde for $30 \mathrm{~min}$ and then permeabilized with $0.5 \%$ Triton X-100 for $20 \mathrm{~min}$. After blocked with $10 \%$ donkey serum in PBS for $1 \mathrm{~h}$, cells were incubated with the primary antibody rabbit anti-Hsp27 (1:100 dilution in PBS, Cell Signaling Technology, 2442) overnight at $4^{\circ} \mathrm{C}$. After thorough washing, cells were incubated with Alexa Fluor 594 donkey anti-rabbit IgG (1:500 in PBS, Invitrogen) at $37^{\circ} \mathrm{C}$ for $1 \mathrm{~h}$. Finally, cells were washed and stained with DAPI. Images were captured using inverted fluorescent microscope (Olympus IX71).

\section{Generation of Hsp27-overexpressed or si- lenced HCC cell lines}

The Hsp27 ORF sequence (NM_001540.3) was

PCR amplified using specific primers and cloned into the lentiviral expression vector pWPXL (Addgene). The specific shRNA sequences of Hsp27 were cloned into the vector PuroGreen (Addgene). Viral packaging was performed by cotransfection of pWPXL, pWPXL-Hsp27, shHsp27\#1 or shHsp27\#2 with packaging plasmid using Lipofectamine 2000 (Invitrogen) in HEK 293T cells. Viruses were harvested at $48 \mathrm{~h}$ after transfection, and viral titers were determined. SK-Hep1, SMMC7721, MHCC97H, and HCCLM3 cells were infected with $1 \times 10^{6}$ recombinant lentivirus-transducing units in the presence of 6 $\mu \mathrm{g} / \mathrm{ml}$ polybrene (Sigma).

\section{CCK8 assay}

Cultured cells were seeded into 96-well plates (1000 per well). Cell Counting Kit-8 assay (Dojindo Laboratories, Kumamoto, Japan) was performed to measure cell viability at different time points according to manufacturer's instructions.

\section{In vitro migration and invasion assays}

Cell migration and invasion were performed as previously described [13].

\section{In vivo metastasis assays}

Tail vein injected models (SK-Hep1 and $\mathrm{MHCC} 97 \mathrm{H})$ were established to validate the role of Hsp27 on HCC metastatic ability in vivo. $2 \times 10^{6}$ indi-

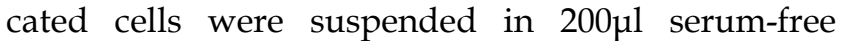
DMEM and injected into the tail vein of nude mice. After five weeks, all of mice were sacrificed. The lung tissues were dissected and fixed with $10 \%$ formalin for at least $24 \mathrm{~h}$. Lung tissues were analyzed by hematoxylin and eosin staining.

\section{Human phospho-kinase array}

Phospho-kinase assays were utilized to analyze alterations of kinase signaling between SK-Hep1-vector and SK-Hep1-Hsp27 cells following the manufacturer's instructions (Proteome Profiler; R\&D Systems, Minneapolis, MN, USA).

\section{PCR array analysis}

The Tumor Metastasis RT2 Profiler PCR Arrays, consisting of 84 genes known to be involved in metastasis, were used to profile MHCC97H-shHsp27, SK-Hep1-Hsp27 cells and their relative control cells as previously described [39].

\section{Gene knockdown using siRNA}

Gene knockdown using siRNA was performed as previously described [13]. siRNAs, including control, MMP2, and ITGA7 were purchased from Biotend (Shanghai, China). The siRNA sequences used were listed in Supplementary Table S2. 


\section{OGX-427 treatment}

Hsp27 ASO (OGX-427) and scrambled control (OGX-411) oligonucleotide were supplied by OncoGenex Technologies. The sequence of OGX-427 corresponds to the human Hsp27 translation initiation site (5'-GGGACGCGGCGCTCGGTCAT-3'). To assess the suppressive effects of Hsp27 inhibitor OGX-427 on metastatic ability of HCC cells in vivo, MHCC97H and HCCLM3 cells $\left(2 \times 10^{6}\right)$ were suspended in $200 \mu l$ serum-free DMEM and injected into the tail vein of nude mice. Then mice were randomly assigned to two groups: OGX-411 or OGX427 group $(20 \mathrm{mg} / \mathrm{kg}$ of OGX-411 or OGX-427 was injected i.p. once daily for 7 days followed by three weekly treatments for another 3 weeks). Five weeks after cell injection, all the mice were sacrificed. The lung tissues were dissected and fixed with $10 \%$ formalin for at least $24 \mathrm{~h}$. Lung tissues were analyzed by hematoxylin and eosin staining and lung metastasis was determined by examining serial sections of every lung tissue block by microscopy. All the mice were manipulated and housed according to protocols approved by the Shanghai Medical Experimental Animal Care Commission.

\section{Statistical analysis}

Statistical analysis was carried out with SPSS software (Chicago, IL). Quantitative variables were analyzed by Student $t$ tests. Kaplan-Meier analysis was used to assess overall survival and tumor recurrence. $P<0.05$ was considered statistically significant.

A Disease Summary for HSPB1

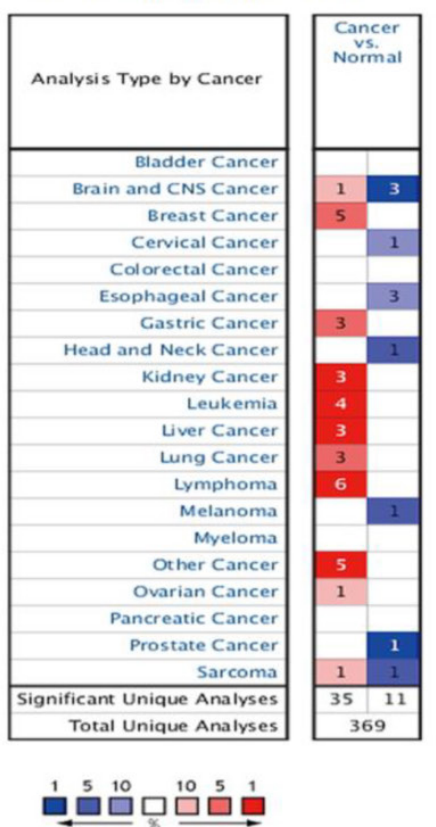

B

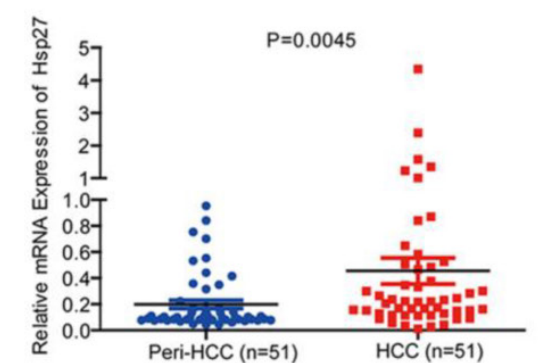

C

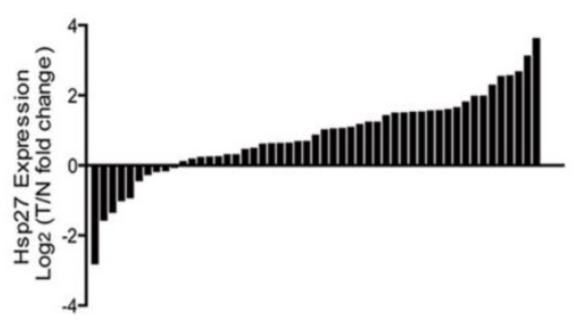

\section{Results}

\section{Clinical significance of Hsp27 overexpression in HCC patients}

Through oncomine data-mining analysis, up-regulation of Hsp27 was found in 11 of 16 cancer types, including breast cancer, liver cancer, gastric cancer, kidney cancer, lung cancer, etc (Fig. 1A). As shown in Fig. 1B-D, Hsp27 transcripts were significantly increased in $60.8 \%$ of HCC tissues $(31 / 51)$ relative to paired noncancerous tissues in patients (cutoff value: ratio of tumor / paired peri-tumor > 1.5). The up-regulation of Hsp27 was also validated by western blotting. (Fig. 1E).

In order to determine the clinical significance of Hsp27 overexpression in HCC, we evaluated the expression of Hsp27 in normal liver cell line and a panel of HCC cell lines. According to western blotting and immunofluorescent staining results, elevated expression of Hsp27 was observed in HCC cell lines with high metastatic potential (Fig. 2A-B). Then, tissue microarray analysis of HCC tissues from 167 patients underwent liver resection was performed. The immunostaining score was evaluated on the basis of percentage score $\times$ intensity score, which is described in Materials and Methods. Levels of Hsp27 protein in tumor tissues were classified as high expression (score ++ and +++$)$ in 81 cases $(81 / 167,48.5 \%)$ and low expression (score + ) or not stained (score -) in 86 cases (86/167, 51.5\%). Representative images of Hsp27 expression were shown in Fig. 2C.

D

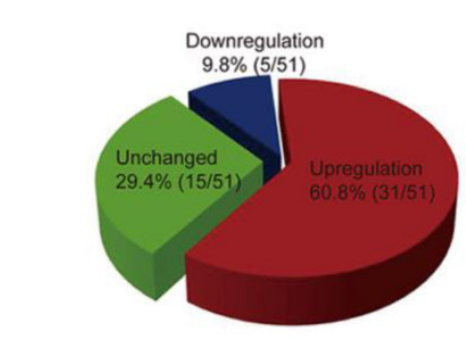

$E$
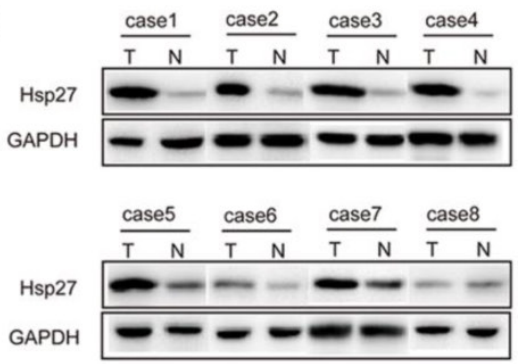

Figure 1. Hsp27 is frequently up-regulated in human HCC. (A) Up-regulation of Hsp27 was found in 11 of 16 cancer types. (B-D) Hsp27 expression in 51 pairs of HCC and noncancerous tissues were detected by real-time PCR. (E) Representative western blot showing the expression of $\mathrm{Hsp} 27$ in tumor and paired noncancerous tissues from eight HCC patients. $* * P<0.01$. 
A
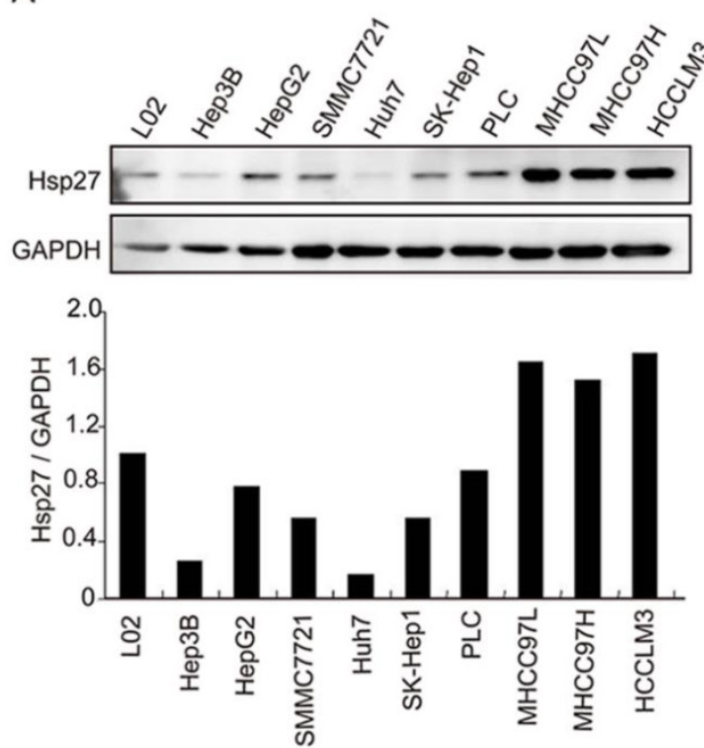

C

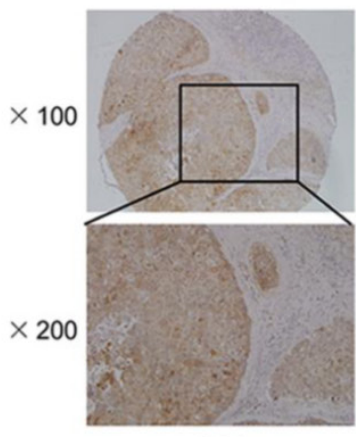

$+++$

D

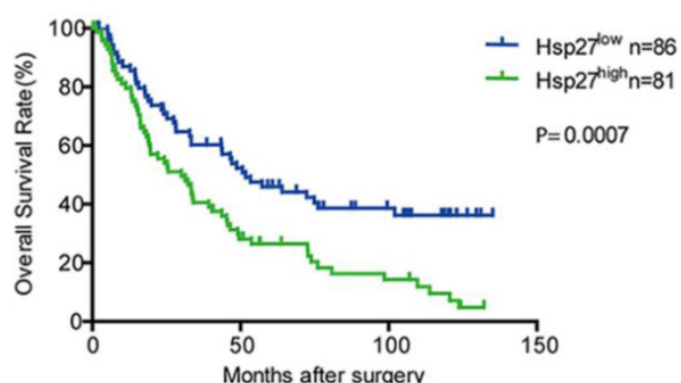

B
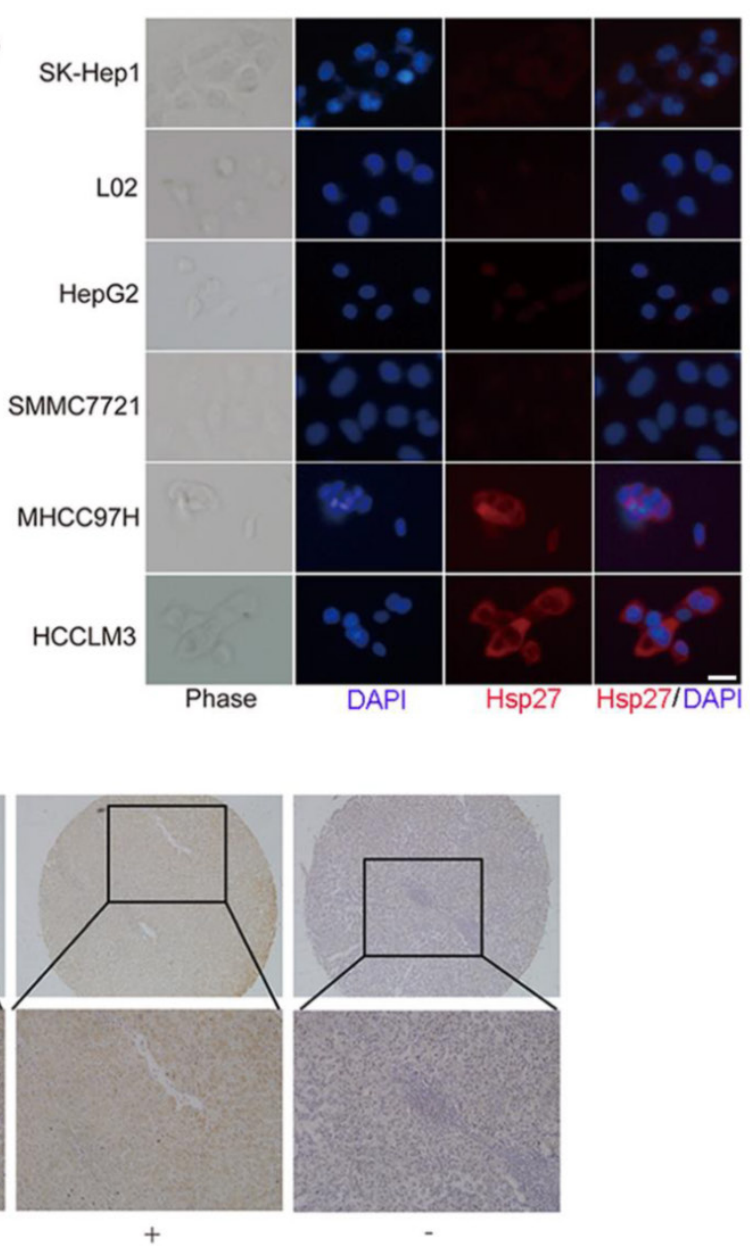

E

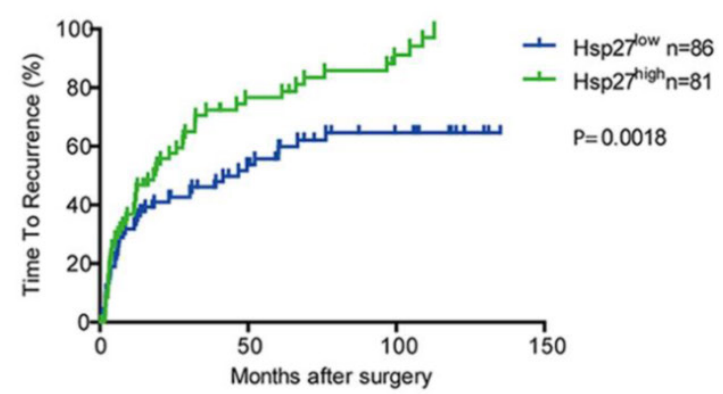

Figure 2. Elevated expression of Hsp27 is associated with poor prognosis in HCC patients. (A-B) Hsp27 expression was measured by western blotting and immunofluorescent analysis (scale bars, $10 \mu \mathrm{m})$ in one human normal liver cell line (LO2) and a panel of HCC cell lines. (C) Typical images showed strong (+++), moderate (++), weak (+), or negative (-) staining of Hsp27 in HCC specimens. (D-E) Kaplan-Meier analysis of overall survival time and time to recurrence in $167 \mathrm{HCC}$ cases based on Hsp27 expression.

Patients with high expression of Hsp27 exhibited worse overall survival (median overall survival time, 30.0 and 51.7 months, respectively; $P<0.001)$ and shorter time to recurrence (median time to recurrence, 18.0 and 46.7 months, respectively; $P<0.01)$ than patients with low expression of Hsp27 (Fig. 2C-E). These data indicated that Hsp27 overexpression could be served as a potential predicting factor for poor prognosis of HCC patients.

\section{Functional role of $\mathrm{Hsp} 27$ in cell migration and invasion}

In order to explore roles of Hsp27 in HCC cell lines, Hsp27 was overexpressed in SK-Hep1 and SMMC-7721 cells through lentiviral infection. Stable overexpression of Hsp27 in these two cell lines was confirmed by real-time PCR and western blotting (Fig. 3A-B). The effect of Hsp27 on cell proliferation was determined by CCK 8 assay. Hsp27 overexpres- 
sion had no significant effect on proliferation of HCC cells (Fig. 3C). However, ectopic expression of Hsp27 significantly facilitated the in vitro migration and invasion of HCC cells (Fig. 3D). Then, we established tail vein injected models to further verify the role of Hsp27 in HCC metastasis. Results from in vivo fluorescence imaging indicated that compared with mice injected with the control cells (metastasis incidence: $1 / 6,16.7 \%)$, mice that received intravenous injection of SK-Hep1-Hsp27 cells showed an obvious increase of metastasis incidence (5/6, 83.3\%; Fig. 3E-F). In addition, the micrometastatic lesions were histologically examined and microscopically detected. The results showed that SK-Hep1-Hsp27 cells caused significant increased numbers of lung metastatic nodules than control cells (Fig. 3G-I). H\&E staining also indicated that several mice bearing SK-Hep1-Hsp27 cells were suffered with liver metastasis (incidence: $2 / 6,33.3 \%$, Fig. 3H).
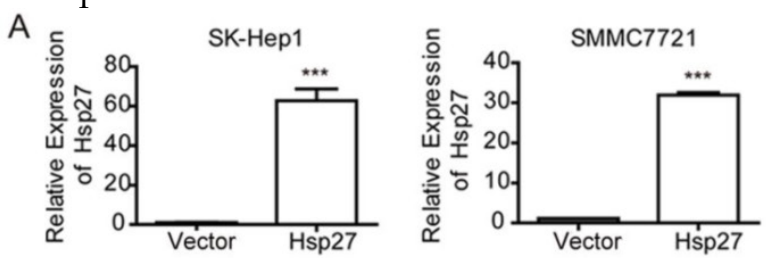

B

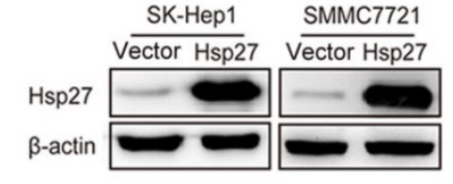

C

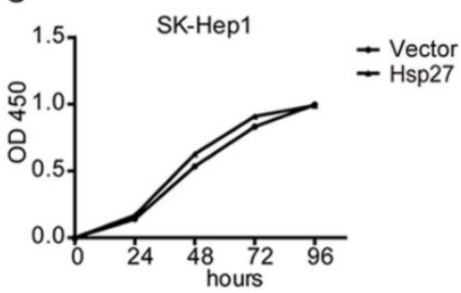

D
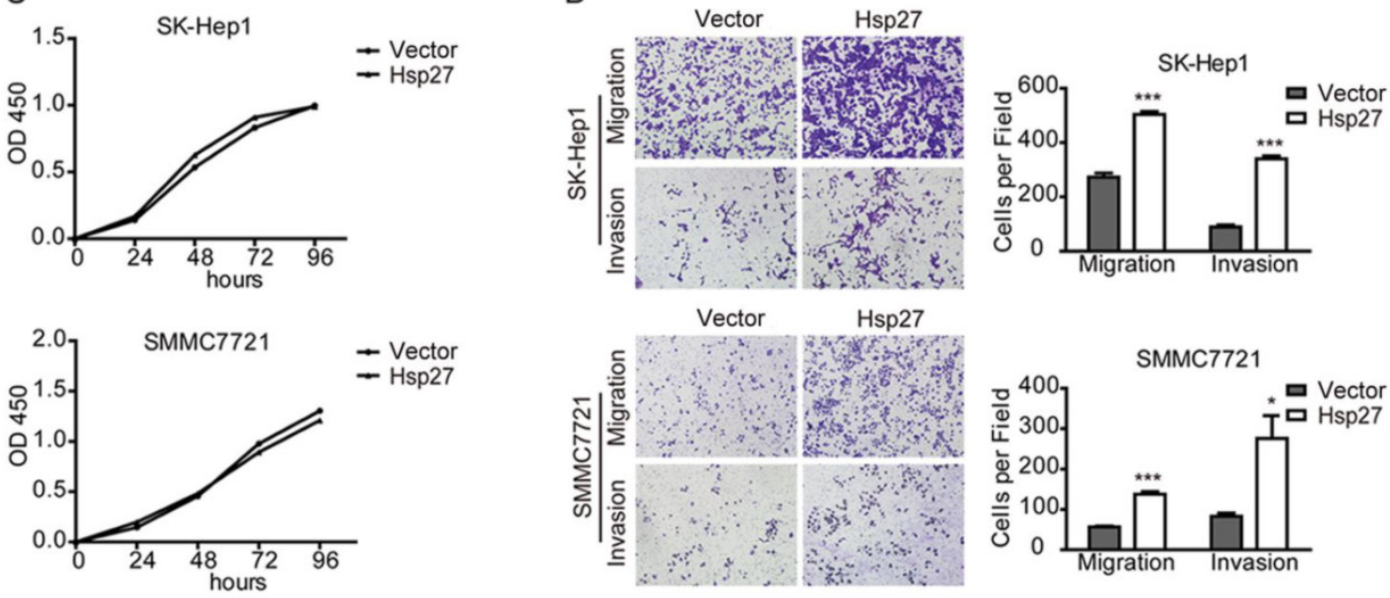

E
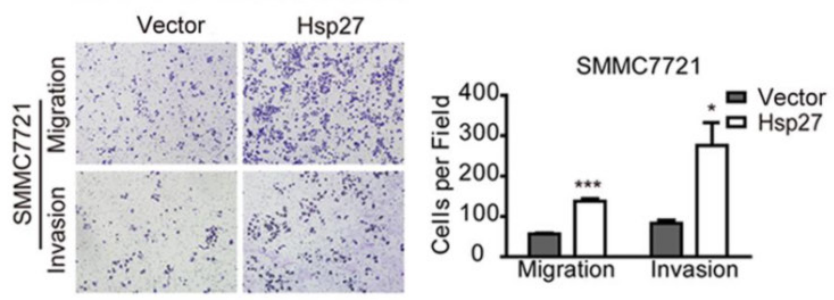

$\mathrm{F}$

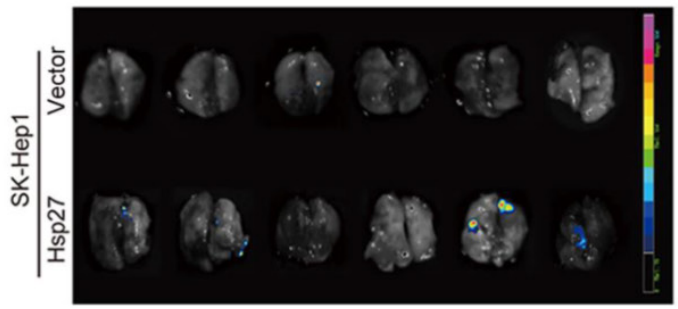

Incidence of lung metastasis in nude mice inoculated

with SK-Hep1 cells through the lateral tail vein

\begin{tabular}{lc}
\hline & Ratio(Lung metastasis/Total) \\
\hline SK-Hep1-Vector & $1 / 6,16.7 \%$ \\
SK-Hep1-Hsp27 & $5 / 6,83.3 \%$
\end{tabular}

G

$\mathrm{H}$

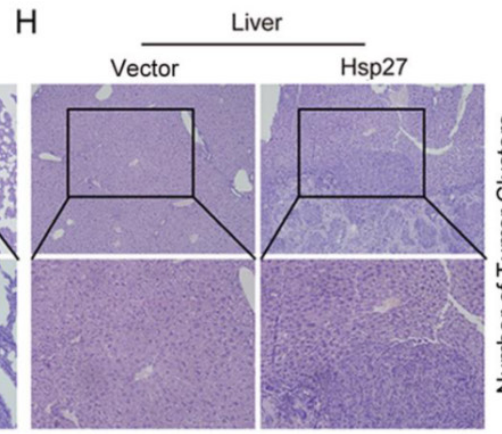

I

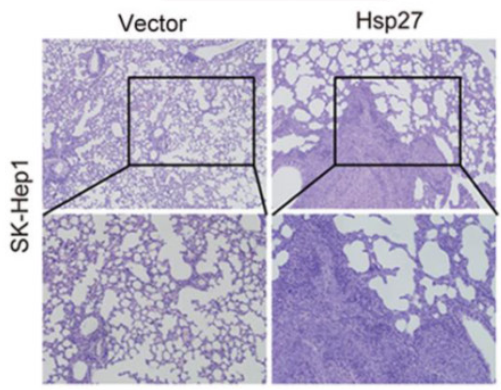

Figure 3. Overexpression of Hsp27 promotes HCC cell migration and invasion. (A-B) The detection of overexpression of Hsp27 in SK-Hepl and SMMC7721 cells by real-time PCR and western blotting. (C) A representative result of the CCK-8 assays for the effects of Hsp27 overexpression on proliferation of SK-Hep1 and SMMC7721 cells. (D) Transwell assays for the effects of Hsp27 overexpression on migratory and invasive potentials of MHCC97H and HCCLM3 cells (magnification, x100). (E-F) Results of in vivo fluorescence imaging and the incidence of lung metastasis in the indicated groups. (G-H) Representative images for lung and liver metastatic nodules in indicated groups (magnification, $\times 100, \times 200$ ). (I) The number of lung metastatic nodules was quantified on serial sections of H\&E staining. $* P<0.05, * * * P<0.001$. 
A

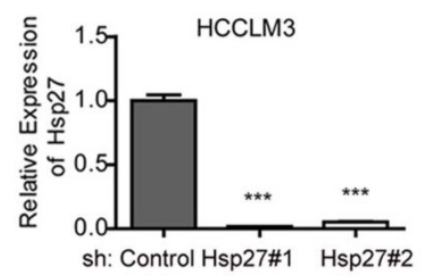

C
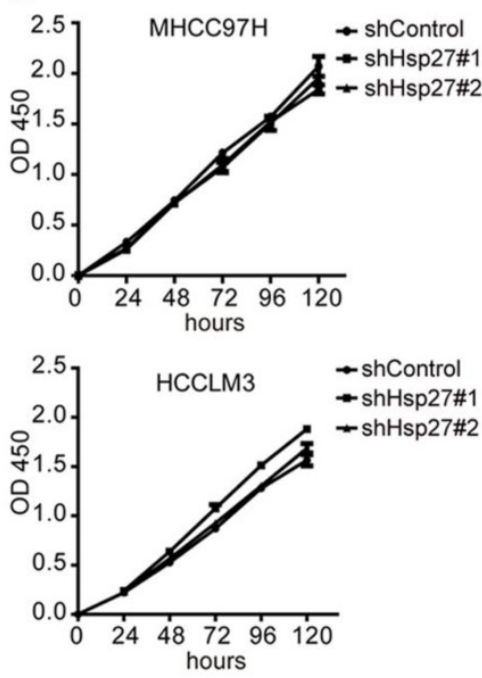

E
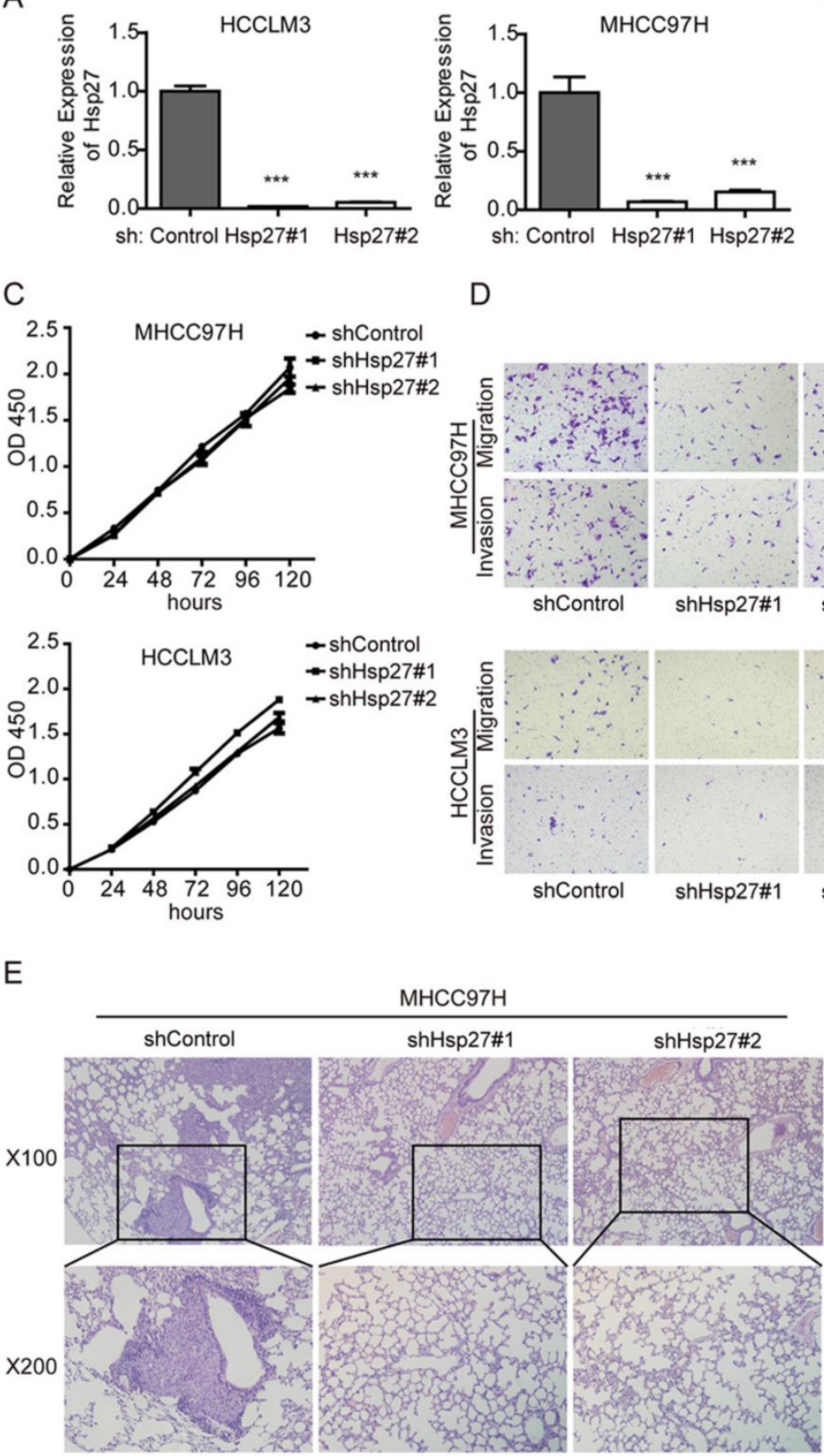

D
B
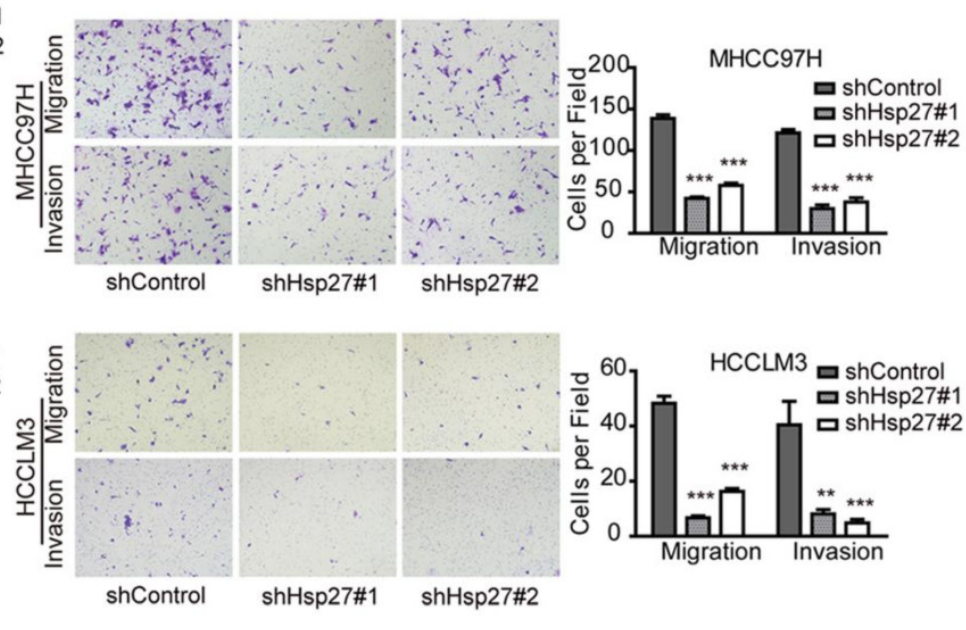

F

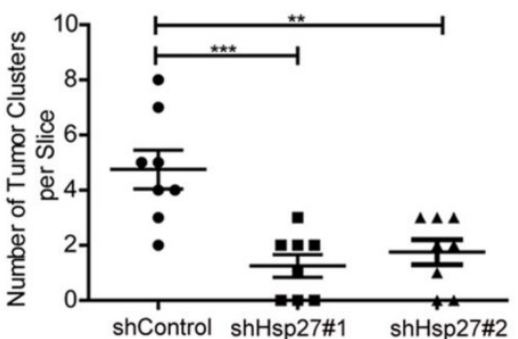

Figure 4. Down-regulation of Hsp27 attenuates HCC cell migration and invasion. (A-B) The detection of shRNA-mediated knockdown of Hsp27 in MHCC97H and HCCLM3 cells by real-time PCR and western blotting. (C) A representative result of the CCK-8 assays for the effects of Hsp27 silence on proliferation of MHCC97H and HCCLM3 cells. (D) Transwell assays for the effects of Hsp27 silence on migratory and invasive potentials of MHCC97H and HCCLM3 cells (magnification, $\times 100$ ). (E) Representative images for metastatic nodules in indicated groups (magnification, $\times 100, \times 200)$. (F) Quantification of metastatic nodules in control and Hsp27-silenced groups. $* * P<$ $0.01, * * * p<0.001$.

To further vadilate the role of Hsp27 in migration and invasion, we employed lentivirus-mediated shRNA knockdown to elucidate the cellular functions of Hsp27. Two independent shRNAs (sh-Hsp27\#1 and sh-Hsp27\#2) showed efficient Hsp27 knockdown in MHCC97H and HCCLM3 cells compared with scrambled shRNA transduced cells (Fig. 4A-B). The effects of Hsp27 knockdown on migration and invasion of MHCC97H and HCCLM3 cells were determined by transwell assays. The results indicated that disruption of Hsp27 expression significantly inhibit cell migration and invasion without affecting cell proliferation (Fig. 4C-D). Furthermore, compared with control cells, Hsp27 knockdown resulted in significant decrease of metastatic foci in MHCC97H cells (Fig. 4E-F).

\section{Akt signaling is responsible for Hsp27-induced cell migration and invasion}

Our studies suggested that Hsp27 functioned as an important regulator in the aggressiveness of HCC cells. In order to identify signaling pathways which functioned as activated downstream of Hsp27, phosphokinase arrays were used to determine alterations 
of kinase signaling between SK-Hep1-Hsp27 and control cells. Four proteins (p38a, AMPKa1, Akt, and JNK) exhibited increased phosphorylation in SK-Hep1-Hsp27 cells (Fig. 5A-B). Then, SK-Hep1-Hsp27 cells were preincubated with inhibitors of p38a, AMPKa1, Akt, or JNK (BIRB796, Dorsomorphin, SP600125, and LY294002) for $24 \mathrm{~h}$. Only LY294002 treatment resulted in an obvious suppression of cell migration and invasion (Fig. 5C and Supplementary Fig. S1). Similar results were obtained using Akt knockdown (Supplementary Fig. S2). Additionally, we confirmed that stable overexpression of Hsp27 led to an increased phosphorylation of Akt in SK-Hep1 cells (Fig. 5D). Knockdown of Hsp27 resulted in reduced phosphorylation of Akt in MHCC97H cells (Fig. 5D). The effects of Hsp27 overexpression on levels of p-Akt in xenograft tumor tissues were also examined by immunohistochemical analysis. Increased level of Hsp27 accompanied with increased phosphorylation of Akt was observed in tumors derived from SK-Hep1-Hsp27 group (Fig. 5E). These findings indicated that Akt pathway may function as downstream signaling of Hsp27-induced aggressiveness in HCC cells.

Furthermore, we analyzed p-Akt in a tissue microarray containing 167 HCC specimens by immunohistochemical analysis. We divided HCC samples into following three groups: high levels of both Hsp27 and p-Akt, either high levels of Hsp27 and p-Akt, and low levels of both Hsp27 and p-Akt. We found that 33 of 176 HCC cases (20.6 \%) exhibited high levels of both Hsp27 and p-Akt. HCC patients with high levels of both Hsp27 and p-Akt showed the worst prognoses $(P$ $<0.01$, Kaplan-Meier analysis, Fig. 5F-H). Conversely, HCC patients who expressed low levels of both Hsp27 and p-Akt had better outcomes (Fig. 5G-H).
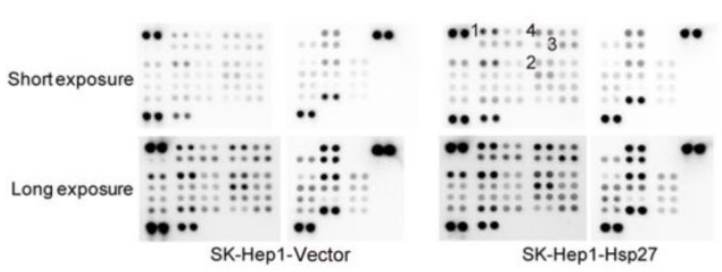

B

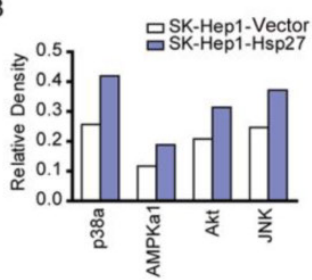

D
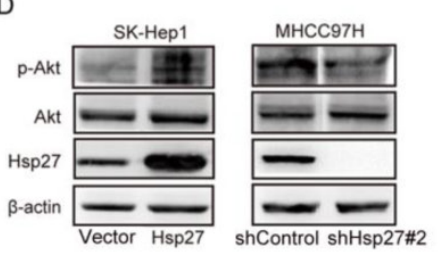

C
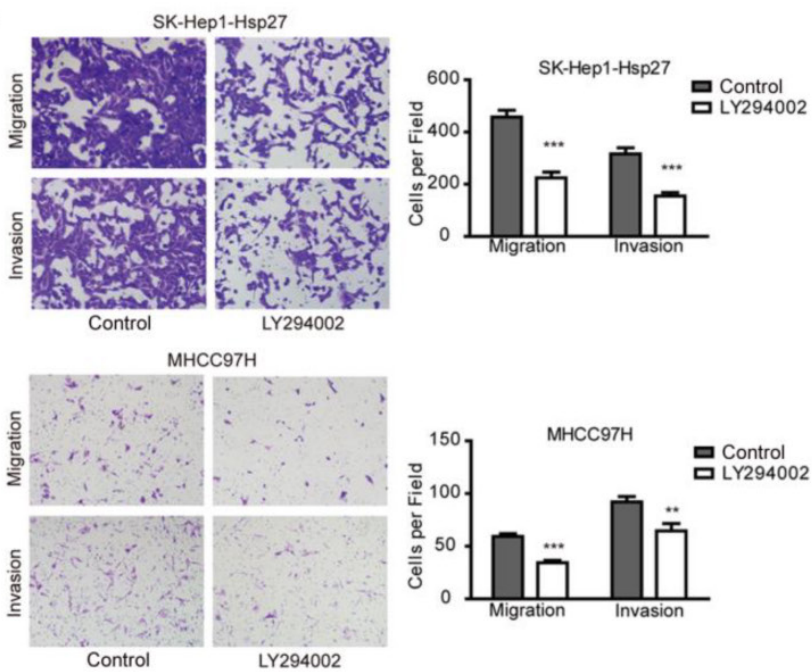

E

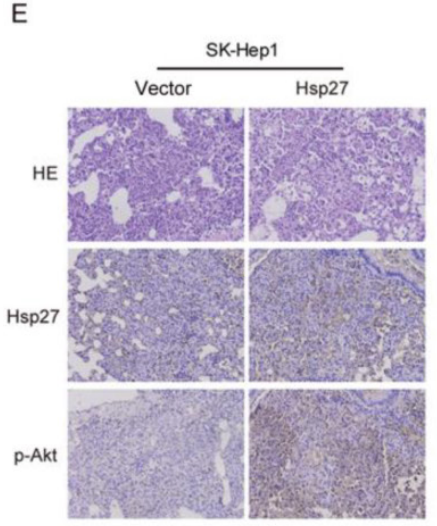

$\mathrm{F}$

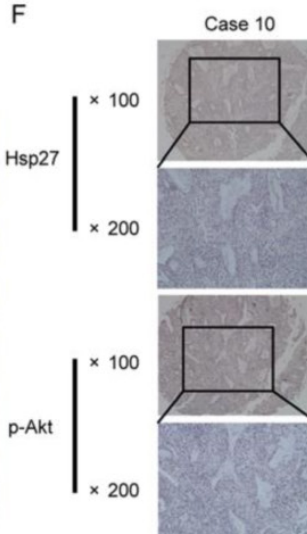

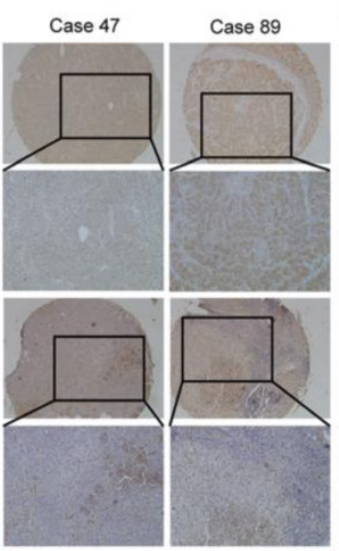
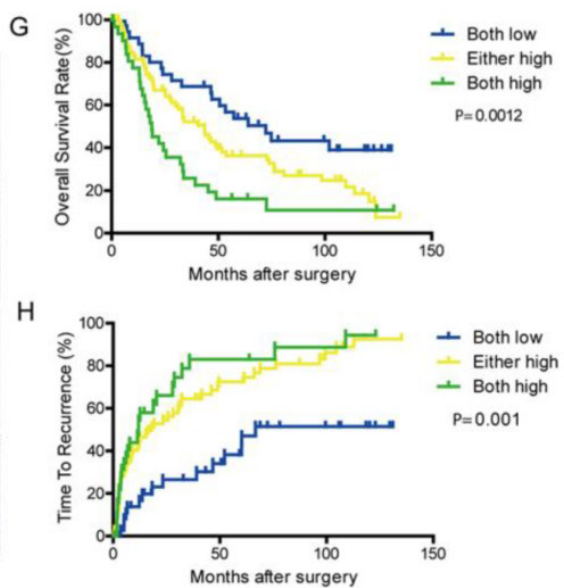

Figure 5. Ectopic expression of Hsp27 leads to increased phosphorylation of Akt. (A-B) Result from phosphokinase arrays suggested that activities of $p 38 \alpha$, AMPKal, Akt, and JNK were increased in SK-Hepl-Hsp27 cells. (C) Migration and matrigel invasion assays were done for SK-Hepl-Hsp27 and MHCC97H cells following treatment with $10 \mu \mathrm{M} \mathrm{LY} 294002$ for $24 \mathrm{~h}$ (magnification, $\times 200$ ). (D) Influence of Hsp27 overexpression or knockdown on the activity of Akt was analyzed by western blotting. (E) Immunohistochemical staining of Hsp27 and p-Akt was performed on serial sections of tumors from SK-Hep1-Hsp27 group and control group (magnification, $\times 200$ ). (F) Representative images of Hsp27 and p-Akt from three patient samples were shown (magnification, $\times 100, \times 200)(\mathbf{G}-\mathbf{H})$ Prognostic values of Hsp27 combined with p-Akt. **P< $0.01, * * * P<0.001$. 


\section{Hsp27 promotes migration and invasion of HCC cells through ITGA7 and MMP2}

To further investigate downstream molecular mechanisms that Hsp27 involved in HCC invasion, Tumor Metastasis RT2 ${ }^{2}$ Profiler PCR Arrays were utilized to compare metastasis-related genes between SK-Hep1-Hsp 27 cells, MHCC97H-sh-Hsp27, and their relative control cells. This analysis revealed a total of 31 up-regulated metastasis-related genes in mRNA levels in SK-Hep1-Hsp27 cells and 9 down-regulated metastasis-related genes in MHCC97H-sh-Hsp27 cells, which had a more than 2-fold change, compared with their control cells respectively (Fig. 6A). Interestingly, among these differential genes, 5 candidate genes exhibited corresponding reverse regulation by Hsp27 overexpression or knockdown (Fig. 6B). Subsequently, these five candidates were validated by qRT-PCR assay (Fig. 6C). To further characterize these genes which were regulated by Akt signaling, we treated SK-Hep1-Hsp 27 and MHCC97H cells with LY294002. Among these five candidates, ITGA7 and MMP2 were regulated by Akt signaling (Fig. 6D and Supplementary Fig. S3). Furthermore, in order to functionally characterize ITGA7 and MMP2, we performed RNA interference studies using SK-Hep1-Hsp 27 and MHCC97H cells (Fig. 6E). Notably, both of ITGA7 and MMP2 consistently regulated the potential of migration and invasion in these two cell lines (Fig. 6F-G). These results suggest that Hsp27 may regulate several clinically and biologically significant genes through ITGA7 and MMP2 during the progression of HCC.
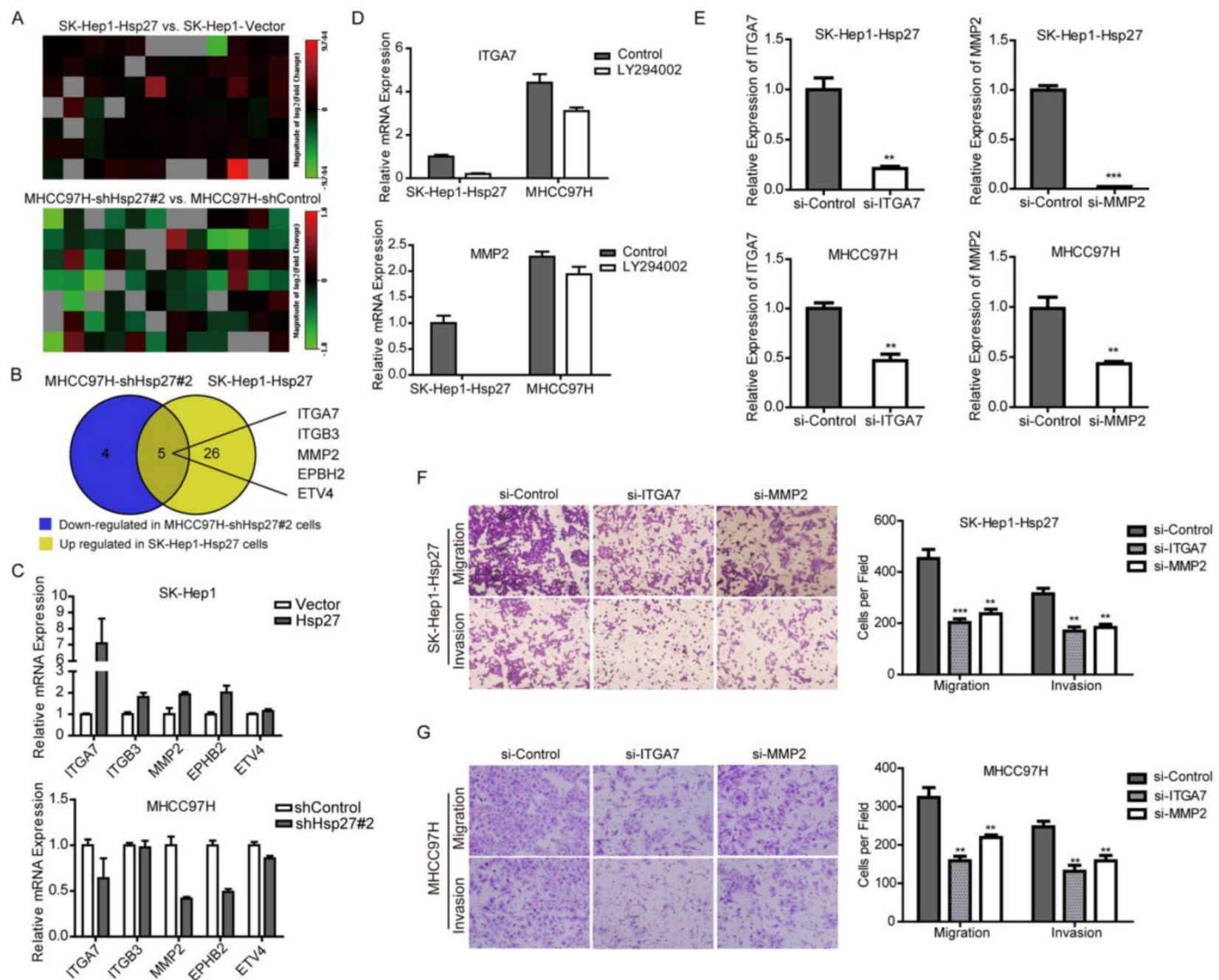

G
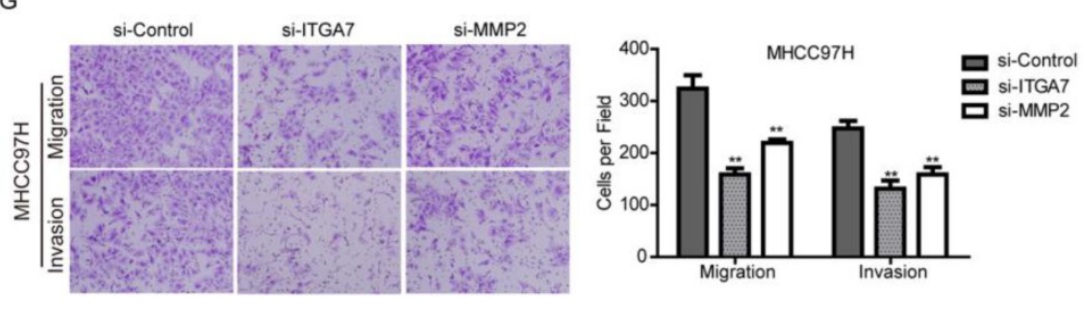

Figure 6. Hsp27 promotes metastatic potential of HCC cells through ITGA7 and MMP2. (A) Tumor Metastasis RT2 Profiler PCR Arrays were utilized to profile metastasis-related genes between SK-Hep1-Hsp27, MHCC97H-sh-Hsp27 cells, and their relative control cells. Heatmaps illustrated alteration of metastasis-related genes in the indicated cells. (B) Five candidate genes were identified with corresponding reverse deregulation by Hsp27 overexpression or knockdown. (C) The detection of mRNA levels of the five candidate genes in SK-Hep1-Hsp27 cells, MHCC97H-sh-Hsp27, and their relative control cells by real-time PCR. (D) The detection of mRNA levels of the candidate genes in SK-Hep1-Hsp27 and MHCC97H cells treated with Akt inhibitor LY294002. (E) siRNA-mediated knockdown of ITGA7 and MMP2 in SK-Hep1-Hsp 27 and MHCC97H cells was analyzed by real-time PCR. (F) Migration and matrigel invasion assays of SK-Hep1-Hsp27 and MHCC97H cells transfected with indicated siRNAs (magnification, $\times 100$ ). 


\section{Hsp27 inhibition using OGX-427 suppresses HCC metastasis}

Having implicated the role of Hsp27 in the aggressiveness of HCC, we next determined whether Hsp27 could be a therapeutic target for HCC. Western blot analysis was performed to evaluate the effect of OGX-427 on Hsp27 expression. OGX-427 obviously down-regulated Hsp27 expression in $\mathrm{MHCC} 97 \mathrm{H}$ and HCCLM3 cells (Fig. 7A).

A
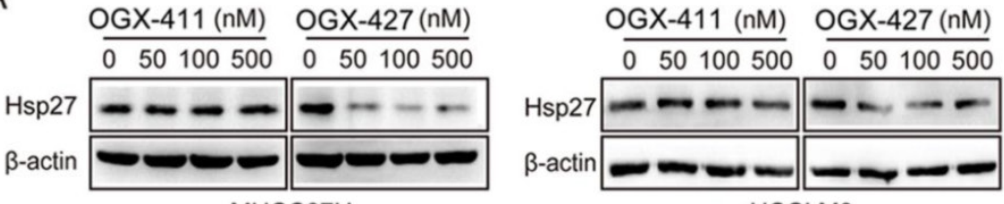

B
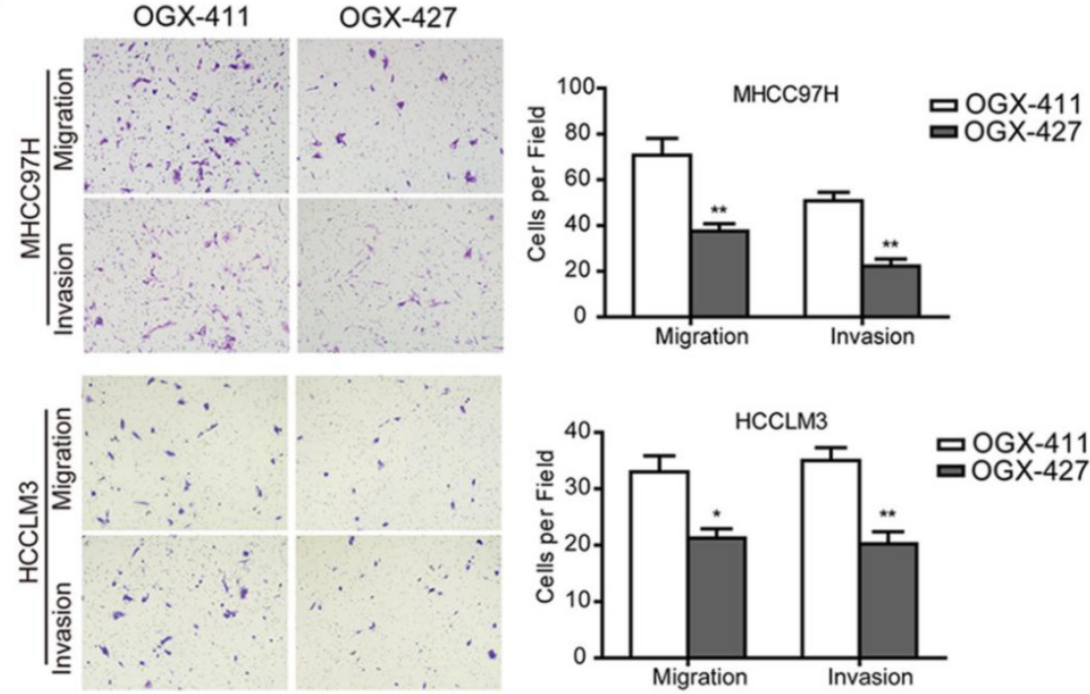

C

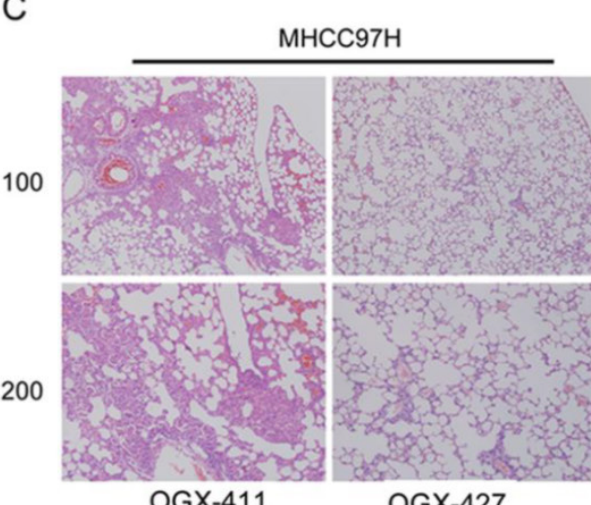

$\mathrm{D}$
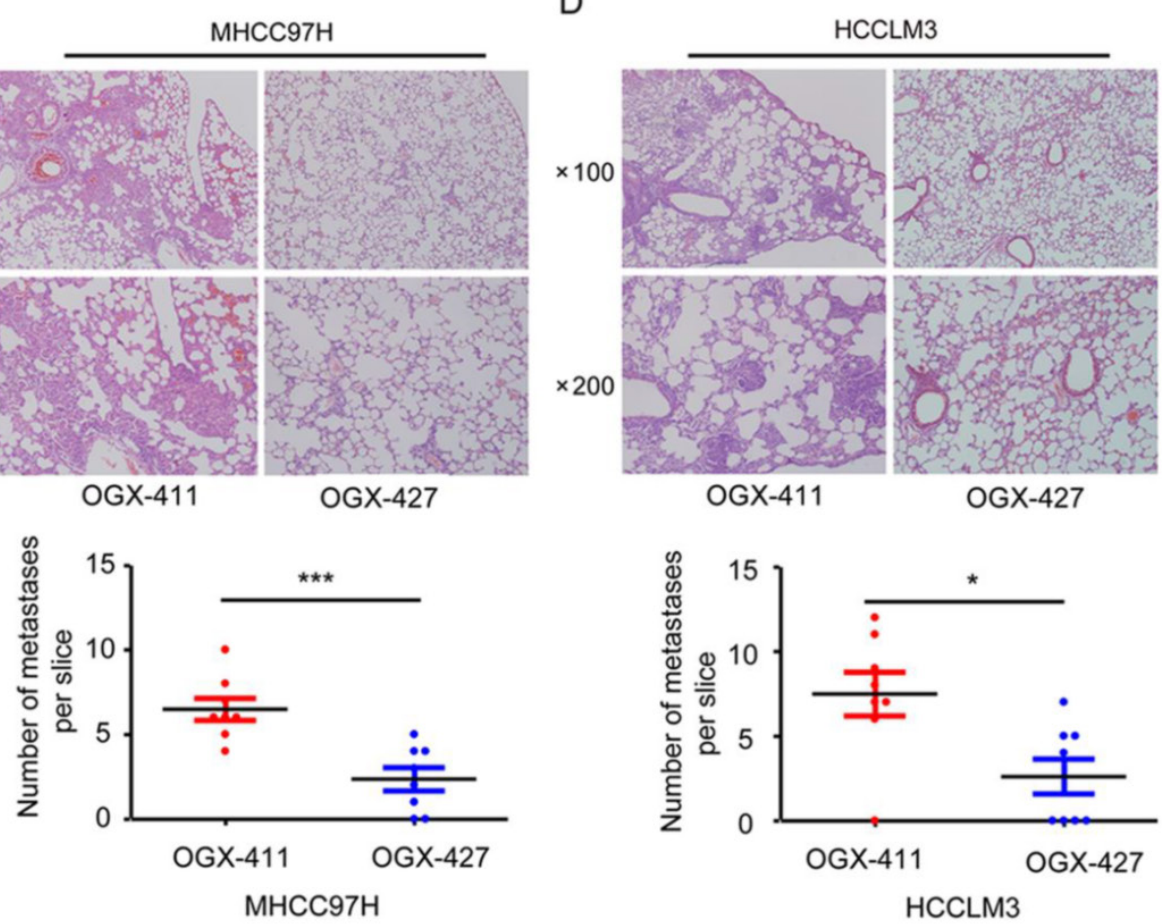

Figure 7. OGX-427 significantly suppresses HCC invasion in vitro and in vivo. (A) MHCC97H and HCCLM3 cells were treated with different concentration of OGX-411 or OGX-427 for $48 \mathrm{~h}$. Whole-cell lysates from indicated cells were analyzed by western blot for Hsp27 and $\beta$-actin. (B) MHCC97H and HCCLM3 cells were treated with $100 \mathrm{nM}$ OGX-411 or OGX-427 for $48 \mathrm{~h}$. Migratory and invasive behaviors were analyzed using transwell and matrigel invasion assays (magnification, $\times 100)$. (C-D) Lung metastasis from mice treated with OGX-411 or OGX-427 were measured through H\&E staining. Representative images for lung metastatic nodules by H\&E staining were shown (magnification, $\times 100, \times 200$ ). The number of lung metastatic nodules was quantified on serial sections of $H \& E$ staining. $* P<0.05, * * P<0.01$. 
Concomitant reductions of migratory and invasive potentials of MHCC97H and HCCLM3 cells were also observed after treatment with OGX-427 (100 nM, Fig. 7B). Furthermore, In order to further determine the role of OGX-427 on HCC metastatic potential in vivo, we established tail vein injected metastatic models using MHCC97H and HCCLM3 cells. For MHCC $97 \mathrm{H}$ cells, compared with OGX-411 group (averaging 6.5 metastases per slice), mice that received OGX-427 showed a $63.5 \%$ reduction in the number of metastatic nodules in the lung, with an average of 2.38 metastases per slice (Fig. 7C). For HCCLM3 cells, OGX-427 significantly reduced number of metastatic nodules with 2.86-fold decrease compared with control group. Collectively, these preclinical evidence suggest the potentiality of Hsp27 as a therapeutic target for HCC metastasis (Fig. 7D).

\section{Discussion}

HCC is a widespread malignancy which deserves intensive investigation. We used clinical database and experimental model to determine Hsp27 as an important regulator for aggressiveness of HCC cells. Mechanistically, Hsp27 promoted the activation of Akt signaling, which subsequently enhanced the expression of ITGA7 and MMP2, and then facilitated invasion and metastasis of HCC cells. These data conclusively indicate that clinically translatable Hsp27 inhibitor OGX427 is a potential therapeutic strategy for HCC metastasis.

High expression of Hsp27 has been reported in several tumors, including breast cancer [18], prostate cancer [15], ovarian cancer [16], and gastric cancer [17], and has been identified as a poor prognostic marker in several tumor types [16,18]. In this study, we evaluated the expression and prognostic relevance of Hsp27 in tissues from HCC patients. In agreement with the results from several other tumor types, the HCC tumor tissue displayed obvious up-regulated expression of Hsp27 compared with noncancerous tissues. A key finding of our study was that Hsp27 expression significantly correlated with worse overall survival and shorter time to recurrence in HCC patients. Overexpression of Hsp27 obviously increased the invasive potential of HCC cell lines in vitro and contributed to distant lung metastasis in vivo. In contrast, shRNA-mediated knockdown of Hsp27 potently inhibited the migration and invasion of HCC cells. These results highlight the potential for exploitation of Hsp27 as a therapeutic target to prevent metastasis and recurrence for HCC patients.

To investigate the underlying mechanisms by which Hsp27 stimulates cell invasion and tumor metastasis, phosphokinase arrays were utilized to identify downstream kinase signaling regulated by Hsp27 in HCC cells. Phospho-antibody microarray is a powerful technology to comprehensively provide novel insight of signaling networks in cancer metastasis. We identified multiple phosphorylated proteins were regulated by Hsp27, including p38a, AMPKa1, JNK, and Akt. p38a MAPK is generally recognized as an upstream molecular of Hsp27. In our study, the activity of p38 MAPK was regulated by Hsp27, which suggested that a positive feedback loop might exist in HCC cell lines [40,41]. It has also been described that AMPK and JNK signalings are involved in cell migration and invasion [42-45]. Among these four differential signaling pathways in our study, only Akt signaling was closely associate with HCC migration and invasion. Furthermore, combination of Hsp27 and p-Akt was a powerful prognostic marker for patients with HCC. Akt signaling is one of the most frequently studied pathways in HCC development and metastasis [46-49]. The underlying mechanisms how Hsp27 contributes to the activation of Akt signaling need to be further identified.

To further demonstrate the downstream molecular mechanism of Hsp27 in invasion and metastasis of HCC, we profiled differentially expressed metastasis-related genes between SK-Hep1-Hsp 27, MHCC97H-sh-Hsp27 cells, and their relative control cells using Tumor Metastasis PCR array. Five candidate genes were identified with reversed alteration after Hsp27 overexpression or knockdown. Among these five candidate genes, ITGA7 and MMP2 were regulated by Akt signaling. A previous study reported that activation of Akt resulted in up-regulation of the Sp1 transcription factor, which led to obvious increase of MMP2 promoter activity in HCC cells [50]. In gastric cancer, MMP2 was regulated by Akt/GSK3 $\beta / \beta$-catenin as well as hypoxia-inducible factor 1a signaling pathways [51]. The mechanism how Akt signaling regulates ITGA7 is still unclear now. In our study, we found that depletion of ITGA7 or MMP2 in HCC cells significantly inhibited Hsp27-mediated invasion. These data suggest that ITGA7 and MMP2 are involved in Hsp27-induced invasion in HCC cells and are potential functional downstream targets of Hsp27.

Hsp27 is an ATP-independent chaperon, so it is not suitable for small-molecule inhibition. However, antisense oligonucleotides or siRNA can be used to suppress the expression of Hsp27, which subsequently generates the effects of chemosensitization, pro-apoptosis and anti-metastasis [10,11]. OGX-427 is a second-generation antisense oligonucleotide that specifically disrupts Hsp27 expression [11]. Phase II clinical trials using OGX-427 in diverse cancers including prostate, ovarian, breast, bladder, and lung have been initiated [29]. OGX-427 has been implicated 
to sensitize cancer cell to multiple targeted drugs (EGFR inhibitors, Hsp90 inhibitor and MET inhibitor), radiation, and some other chemotherapeutics [16, 24-29]. In addition, Hsp27 inhibition by OGX-427 reduced tumor metastasis in a murine model of prostate cancer [36]. Hsp27 knockdown also promoted Snail proteasomal degradation, thus inhibiting TGF- $\beta 1$-induced EMT [52]. In a phase I clinical trial, OGX-427 treatment decreased circulating tumor cells number in prostate cancer patients.[36] In our study, we demonstrate that OGX-427 could effectively inhibit cell invasion in vitro and inhibit distant lung metastasis in vivo. It is the first time we present the evidence that Hsp27 may service as a therapeutic target for HCC metastasis. As we described above, Hsp27 overexpression can be served as a potential predicting factor for poor prognosis of HCC patients. And we suppose that these patients will be more suitable for Hsp27 targeted therapy. Pathological classification based on Hsp27 expression may be required to predict which HCC patients will be benefited from OGX-427 treatment.

In summary, our study indicates that Hsp27 contributes to HCC progression by activation of Akt signaling and enhances expression of ITGA7 and MMP2. Hsp27 expression alone or in combination with p-Akt serves as a novel prognostic indicator for HCC patients. Targeting Hsp27 with OGX-427 may hold attractive therapeutic promise for the improvement of outcomes for HCC patients, especially for the HCC patients who have increased expression of Hsp27.

\section{Supplementary Material}

Supplementary Figures S1-S3 and Tables S1-S2. http://www.thno.org/v06p0558s1.pdf

\section{Abbreviations}

HCC, hepatocellular carcinoma; Hsps, heat shock proteins; TCTP, translationally controlled tumor protein; OS, overall survival; TTR, time to recurrence.

\section{Acknowledgements}

This work was supported by grants from National Key Basic Research Program of China (973 Program: 2015CB553905), National Natural Science Foundation of China (81301818, 81402278, 81572311, and 81421001), National Key Sci-Tech Special Project of China (2012ZX10002011-004), and projects of Special Research Fund for Healthy (201402003), Shanghai Jiao Tong University School of Medicine (YG2014MS44 and YG2015QN34), State Key Laboratory of Oncogenes and Related Genes (SB16-04) and Key Discipline and Specialty Foundation of Shanghai
Municipal Commission of Health and Family Planning.

\section{Competing Interests}

The authors have declared that no competing interest exists.

\section{References}

1. Torre LA, Bray F, Siegel RL, Ferlay J, Lortet-Tieulent J, Jemal A. Global cancer statistics, 2012. CA Cancer J Clin. 2015; 65: 87-108.

2. Berasain C. Hepatocellular carcinoma and sorafenib: too many resistance mechanisms? Gut. 2013; 62: 1674-5.

3. Llovet JM, Di Bisceglie AM, Bruix J, Kramer BS, Lencioni R, Zhu AX, et al. Design and endpoints of clinical trials in hepatocellular carcinoma. J Natl Cancer Inst. 2008; 100: 698-711.

4. Ji J, Shi J, Budhu A, Yu Z, Forgues M, Roessler S, et al. MicroRNA expression, survival, and response to interferon in liver cancer. N Engl J Med. 2009; 361: 1437-47.

5. Portolani N, Coniglio A, Ghidoni S, Giovanelli M, Benetti A, Tiberio GA, et al. Early and late recurrence after liver resection for hepatocellular carcinoma: prognostic and therapeutic implications. Ann Surg. 2006; 243: 229-35.

6. Forner A, Llovet JM, Bruix J. Hepatocellular carcinoma. Lancet. 2012; 379: 1245-55.

7. Llovet JM, Ricci S, Mazzaferro V, Hilgard P, Gane E, Blanc JF, et al. Sorafenib in advanced hepatocellular carcinoma. N Engl J Med. 2008; 359: 378-90.

8. Gauthier A, Ho M. Role of sorafenib in the treatment of advanced hepatocellular carcinoma: An update. Hepatol Res. 2013; 43: 147-54.

9. Akerfelt M, Morimoto RI, Sistonen L. Heat shock factors: integrators of cell stress, development and lifespan. Nat Rev Mol Cell Biol. 2010; 11: 545-55.

10. Azad AA, Zoubeidi A, Gleave ME, Chi KN. Targeting heat shock proteins in metastatic castration-resistant prostate cancer. Nat Rev Urol. 2015; 12: 26-36.

11. Ischia J, So AI. The role of heat shock proteins in bladder cancer. Nat Rev Urol. 2013; 10: 386-95.

12. Wang C, Jiang K, Gao D, Kang X, Sun C, Zhang Q, et al. Clusterin protects hepatocellular carcinoma cells from endoplasmic reticulum stress induced apoptosis through GRP78. PLoS One. 2013; 8: e55981.

13. Wang C, Jin G, Jin H, Wang N, Luo Q, Zhang Y, et al. Clusterin facilitates metastasis by EIF3I/Akt/MMP13 signaling in hepatocellular carcinoma. Oncotarget. 2015; 6: 2903-16

14. Wang C, Jiang K, Kang X, Gao D, Sun C, Li Y, et al. Tumor-derived secretory clusterin induces epithelial-mesenchymal transition and facilitates hepatocellular carcinoma metastasis. Int J Biochem Cell Biol. 2012; 44: 2308-20.

15. Bubendorf L, Kolmer M, Kononen J, Koivisto P, Mousses S, Chen Y, et al. Hormone therapy failure in human prostate cancer: analysis by complementary DNA and tissue microarrays. J Natl Cancer Inst. 1999; 91: 1758-64.

16. Arts HJ, Hollema H, Lemstra W, Willemse PH, De Vries EG, Kampinga HH, et al. Heat-shock-protein-27 (hsp27) expression in ovarian carcinoma: relation in response to chemotherapy and prognosis. Int J Cancer. 1999; 84: 234-8.

17. Ehrenfried JA, Herron BE, Townsend CJ, Evers BM. Heat shock proteins are differentially expressed in human gastrointestinal cancers. Surg Oncol. 1995; 4: 197-203.

18. Storm FK, Mahvi DM, Gilchrist KW. Heat shock protein 27 overexpression in breast cancer lymph node metastasis. Ann Surg Oncol. 1996; 3: 570-3.

19. Baylot V, Katsogiannou M, Andrieu C, Taieb D, Acunzo J, Giusiano S, et al. Targeting TCTP as a new therapeutic strategy in castration-resistant prostate cancer. Mol Ther. 2012; 20: 2244-56.

20. Bruey JM, Ducasse C, Bonniaud P, Ravagnan L, Susin SA, Diaz-Latoud C, et al. Hsp27 negatively regulates cell death by interacting with cytochrome c. Nat Cell Biol. 2000; 2: 645-52.

21. Voss OH, Batra S, Kolattukudy SJ, Gonzalez-Mejia ME, Smith JB, Doseff AI. Binding of caspase- 3 prodomain to heat shock protein 27 regulates monocyte apoptosis by inhibiting caspase-3 proteolytic activation. J Biol Chem. 2007; 282: 25088-99.

22. Zhao GY, Ding JY, Lu CL, Lin ZW, Guo J. The overexpression of 14-3-3zeta and Hsp27 promotes non-small cell lung cancer progression. Cancer. 2014; 120: $652-63$

23. Katsogiannou M, Andrieu C, Baylot V, Baudot A, Dusetti NJ, Gayet O, et al. The functional landscape of Hsp27 reveals new cellular processes such as DNA repair and alternative splicing and proposes novel anticancer targets. Mol Cell Proteomics. 2014; 13: 3585-601.

24. Lelj-Garolla B, Kumano M, Beraldi E, Nappi L, Rocchi P, Ionescu DN, et al. Hsp27 Inhibition with OGX-427 Sensitizes Non-Small Cell Lung Cancer Cells to Erlotinib and Chemotherapy. Mol Cancer Ther. 2015; 14: 1107-16.

25. Hadchity E, Aloy MT, Paulin C, Armandy E, Watkin E, Rousson R, et al. Heat shock protein 27 as a new therapeutic target for radiation sensitization of head and neck squamous cell carcinoma. Mol Ther. 2009; 17: 1387-94.

26. Lamoureux F, Thomas C, Yin MJ, Fazli L, Zoubeidi A, Gleave ME. Suppression of heat shock protein 27 using OGX-427 induces endoplasmic reticulum stress and potentiates heat shock protein 90 inhibitors to delay castrate-resistant prostate cancer. Eur Urol. 2014; 66: 145-55. 
27. Musiani D, Konda JD, Pavan S, Torchiaro E, Sassi F, Noghero A, et al. Heat-shock protein 27 (HSP27, HSPB1) is up-regulated by MET kinase inhibitors and confers resistance to MET-targeted therapy. FASEB J. 2014; 28: 4055-67.

28. Kamada M, So A, Muramaki M, Rocchi P, Beraldi E, Gleave M. Hsp27 knockdown using nucleotide-based therapies inhibit tumor growth and enhance chemotherapy in human bladder cancer cells. Mol Cancer Ther. 2007; 6: 299-308.

29. Baylot V, Andrieu C, Katsogiannou M, Taieb D, Garcia S, Giusiano S, et al. OGX-427 inhibits tumor progression and enhances gemcitabine chemotherapy in pancreatic cancer. Cell Death Dis. 2011; 2: e221.

30. Kang S, Elf S, Lythgoe K, Hitosugi T, Taunton J, Zhou W, et al. p90 ribosomal S6 kinase 2 promotes invasion and metastasis of human head and neck squamous cell carcinoma cells. J Clin Invest. 2010; 120: 1165-77.

31. Yang F, Yin Y, Wang F, Wang Y, Zhang L, Tang Y, et al. miR-17-5p Promotes migration of human hepatocellular carcinoma cells through the p38 mitogen-activated protein kinase-heat shock protein 27 pathway. Hepatology. 2010; 51: 1614-23.

32. Wei L, Liu TT, Wang HH, Hong HM, Yu AL, Feng HP, et al. Hsp27 participates in the maintenance of breast cancer stem cells through regulation of epithelial-mesenchymal transition and nuclear factor-kappaB. Breast Cancer Res. 2011; 13: R101.

33. Gibert B, Eckel B, Gonin V, Goldschneider D, Fombonne J, Deux B, et al. Targeting heat shock protein 27 (HspB1) interferes with bone metastasis and tumour formation in vivo. Br J Cancer. 2012; 107: 63-70.

34. Zhu Z, Xu X, Yu Y, Graham M, Prince ME, Carey TE, et al. Silencing heat shock protein 27 decreases metastatic behavior of human head and neck squamous cell cancer cells in vitro. Mol Pharm. 2010; 7: 1283-90.

35. Cordonnier T, Bishop JL, Shiota M, Nip KM, Thaper D, Vahid S, et al. Hsp27 regulates EGF/beta-catenin mediated epithelial to mesenchymal transition in prostate cancer. Int J Cancer. 2015; 136: E496-507.

36. Shiota M, Bishop JL, Nip KM, Zardan A, Takeuchi A, Cordonnier T, et al. Hsp27 regulates epithelial mesenchymal transition, metastasis, and circulating tumor cells in prostate cancer. Cancer Res. 2013; 73: 3109-19.

37. Pavan S, Musiani D, Torchiaro E, Migliardi G, Gai M, Di Cunto F, et al. HSP27 is required for invasion and metastasis triggered by hepatocyte growth factor. Int J Cancer. 2014; 134: 1289-99.

38. Guo K, Liu $Y$, Zhou $H$, Dai $Z$, Zhang J, Sun $R$, et al. Involvement of protein kinase $C$ beta-extracellular signal-regulating kinase $1 / 2 /$ p38 mitogen-activated protein kinase-heat shock protein 27 activation in hepatocellular carcinoma cell motility and invasion. Cancer Sci. 2008; 99: 486-96.

39. Luo Q, Wang C, Jin G, Gu D, Wang N, Song J, et al. LIFR functions as a metastasis suppressor in hepatocellular carcinoma by negatively regulating phosphoinositide 3-kinase/AKT pathway. Carcinogenesis. 2015; 36: 1201-12.

40. Xu L, Chen S, Bergan RC. MAPKAPK2 and HSP27 are downstream effectors of p38 MAP kinase-mediated matrix metalloproteinase type 2 activation and cell invasion in human prostate cancer. Oncogene. 2006; 25: 2987-98.

41. Lin SP, Lee YT, Wang JY, Miller SA, Chiou SH, Hung MC, et al. Survival of cancer stem cells under hypoxia and serum depletion via decrease in PP2A activity and activation of p38-MAPKAPK2-Hsp27. PLoS One. 2012; 7: e49605.

42. Cerezo M, Tichet M, Abbe P, Ohanna M, Lehraiki A, Rouaud F, et al. Metformin blocks melanoma invasion and metastasis development in AMPK/p53-dependent manner. Mol Cancer Ther. 2013; 12: 1605-15.

43. Li N, Huang D, Lu N, Luo L. Role of the LKB1/AMPK pathway in tumor invasion and metastasis of cancer cells (Review). Oncol Rep. 2015: 34: 2821-6.

44. Pallavi SK, Ho DM, Hicks C, Miele L, Artavanis-Tsakonas S. Notch and Mef2 synergize to promote proliferation and metastasis through JNK signal activation in Drosophila. EMBO J. 2012; 31: 2895-907.

45. Tong X, Barbour M, Hou K, Gao C, Cao S, Zheng J, et al. Interleukin-33 predicts poor prognosis and promotes ovarian cancer cell growth and metastasis through regulating ERK and JNK signaling pathways. Mol Oncol. 2016; 10: 113-25.

46. Liu L, Dai Y, Chen J, Zeng T, Li Y, Chen L, et al. Maelstrom promotes hepatocellular carcinoma metastasis by inducing epithelial-mesenchymal transition by way of Akt/GSK-3beta/Snail signaling. Hepatology. 2014; 59: 531-43.

47. Chen J, Chan AW, To KF, Chen W, Zhang Z, Ren J, et al. SIRT2 overexpression in hepatocellular carcinoma mediates epithelial to mesenchymal transition by protein kinase $\mathrm{B} /$ glycogen synthase kinase-3beta/beta-catenin signaling. Hepatology. 2013; 57: 2287-98.

48. Wen W, Ding J, Sun W, Fu J, Chen Y, Wu K, et al. Cyclin G1-mediated epithelial-mesenchymal transition via phosphoinositide 3-kinase/Akt signaling facilitates liver cancer progression. Hepatology. 2012; 55: 1787-98.

49. Fu J, Chen Y, Cao J, Luo T, Qian YW, Yang W, et al. p28GANK overexpression accelerates hepatocellular carcinoma invasiveness and metastasis via phosphoinositol 3-kinase/AKT/hypoxia-inducible factor-1alpha pathways. Hepatology. 2011; 53: 181-92.

50. Sze KM, Wong KL, Chu GK, Lee JM, Yau TO, Ng IO. Loss of phosphatase and tensin homolog enhances cell invasion and migration through AKT/Sp-1 transcription factor/matrix metalloproteinase 2 activation in hepatocellular carcinoma and has clinicopathologic significance. Hepatology. 2011; 53: 1558-69.

51. Tsai CY, Wang CS, Tsai MM, Chi HC, Cheng WL, Tseng $\mathrm{YH}$, et al. Interleukin-32 increases human gastric cancer cell invasion associated with tumor progression and metastasis. Clin Cancer Res. 2014; 20: 2276-88.
52. Wettstein G, Bellaye PS, Kolb M, Hammann A, Crestani B, Soler P, et al. Inhibition of HSP27 blocks fibrosis development and EMT features by promoting Snail degradation. FASEB J. 2013; 27: 1549-60. 